\title{
A COMPLETE SYSTEM FOR THE SIMPLE GROUP $G_{60}^{6}$
}

\author{
BY C. W. STROM
}

The problem of this paper is to obtain an irreducible set of polynomials in terms of which all the polynomials that are invariant under the simple group $G_{60}^{6}$ can be expressed as polynomial functions.

No polynomials of degrees and extents 1 and 2 respectively are invariant under this group except the respective elementary symmetric polynomials, $E_{1}$ and $E_{2}$. Hence, this irreducible set will contain no polynomials of extents 1 and 2 other than the elementary symmetric polynomials which we shall write as $S$-polynomials :

$$
S_{1} \equiv E_{1} \quad \text { and } \quad S_{2} \equiv E_{2} .
$$

The group leaves invariant the set of triples,

$$
123,134,145,156,162,235,346,452,563,624 \text {, }
$$

and the complementary set

$$
124,146,163,135,152,243,465,632,354,526 .
$$

Hence the $S$-polynomials,

$$
\begin{aligned}
& S_{123} \equiv x_{1} x_{2} x_{3}+x_{1} x_{3} x_{4}+x_{1} x_{4} x_{5}+\cdots, \\
& S_{124} \equiv x_{1} x_{2} x_{4}+x_{1} x_{4} x_{6}+x_{1} x_{6} x_{3}+\cdots,
\end{aligned}
$$

or, as we shall write them,

$$
\begin{aligned}
& S_{123} \equiv 123+134+145+\cdots, \\
& S_{124} \equiv 124+146+163+\cdots,
\end{aligned}
$$

are invariant under the group. Clearly, the polynomials $S_{1 i 2 i 3 k}$ and $S_{1 i^{2 j} 4_{k}}$ are also invariant under the group, where

$$
S_{1^{i} i_{i 3 k}}+S_{1^{i} i^{i 4 k}} \equiv \Sigma_{1^{i 2 i 3 k}}
$$

the general symmetric polynomial of extent 3 on 6 variables.

Each of the 15 quadruples that can be selected from among the numbers $1, \cdots, 6$ may be regarded as the intersection of two of the triples in each of the sets above. Thus, 1234 is the 
intersection of 123 and 134 in the first set and of 124 and 234 in the second set. We shall write the quadruple 1234 in the form $13 \cdot 24$ and we shall define the $S$-polynomial of extent 4 and degree 4 :

$$
\begin{aligned}
S_{13 \cdot 24} \equiv & 13 \cdot 24+23 \cdot 45+12 \cdot 36+45 \cdot 12+26 \cdot 14+16 \cdot 25 \\
& +14 \cdot 35+34 \cdot 16+56 \cdot 13+15 \cdot 46+25 \cdot 34+35 \cdot 26 \\
& +24 \cdot 56+36 \cdot 45+46 \cdot 23 .
\end{aligned}
$$

Then, $S_{13 \cdot 24} \equiv E_{4}$, but

$$
\begin{aligned}
\Sigma_{1^{i} 2^{i} 3_{4} l} \equiv & S_{1^{i} 3 i \cdot 2^{k} 4 l}+S_{1^{i} 3^{k} \cdot 2 i 4 l}+S_{1^{i} 3 l \cdot 2 i 4 k} \\
& +S_{1^{i} 3^{k} \cdot 2 i 4 l}+S_{1^{i} 3 l \cdot 2 i 4 k}+S_{1 k 3 l \cdot 2 i 4 i} .
\end{aligned}
$$

Each of the six quintuples that can be selected from among the numbers $1, \cdots, 6$ contains ten triples and these form five pairs, consisting of one triple from each set having an element in common. Thus, the quintuple 12345 contains the following pairs:

513,$524 ; 423,415 ; 345,321 ; 214,253$; and 125, 143.

We define the $S$-polynomial of extent 5 and degree 5 : $S_{5 \cdot 13 \cdot 24} \equiv 5 \cdot 13 \cdot 24+4 \cdot 23 \cdot 15+3 \cdot 45 \cdot 32+2 \cdot 14 \cdot 53+1 \cdot 25 \cdot 43$ + terms obtained from the five remaining quintuples. We define further

$$
\begin{aligned}
& S_{5^{i} \cdot 1^{i} 3^{k} \cdot 24^{m}} \equiv 5^{i} \cdot 1^{j} 3^{k} \cdot 2^{l} 4^{m}+5^{i} \cdot 1^{k} 3^{j} \cdot 2^{m} 4^{l}+4^{i} \cdot 2^{i} 3^{k} \cdot 1^{l} 5^{m} \\
& +4^{i} \cdot 2^{k} 3^{j} \cdot 1^{m} 5^{l}+3^{i} \cdot 4^{j} 5^{k} \cdot 2^{l} 1^{m}+3^{i} \cdot 4^{k} 5^{j} \cdot 2^{m} 1^{l} \\
& +2^{i} \cdot 1^{j} 4^{k} \cdot 5^{l} 3^{m}+2^{i} \cdot 1^{k} 4^{j} \cdot 5^{m} 3^{l}+1^{i} \cdot 2^{j} 5^{k} \cdot 4^{l} 3^{m} \\
& +1^{i} \cdot 2^{k} 5^{j} \cdot 4^{m} 3^{l}+50 \text { additional terms, }
\end{aligned}
$$

where the additional terms are obtained from the 5 remaining quintuples. Then

and

$$
S_{5 \cdot 13 \cdot 24} \equiv 5 E_{5},
$$

$$
\begin{aligned}
& 5 \Sigma_{1^{i} 2^{i} 3^{k} l l^{m}} \equiv S_{5 i \cdot 1 i 3^{k} \cdot 2 l_{4}^{m}}+S_{5 i \cdot 1^{i} 3^{k} \cdot 2^{m}{ }^{\prime} l}+S_{5 \imath \cdot 1 i 3 l \cdot 2^{k} 4^{m}} \\
& +S_{5^{i} \cdot 1 i 3 l \cdot 2^{m} 4^{k}}+S_{5 i \cdot 13^{m} \cdot 2^{k} 4 l}+S_{5 i \cdot 1 i 3^{m} \cdot 2 l 4^{k}}+S_{5 i \cdot 1 k 3 l \cdot 2 i 4^{m}}
\end{aligned}
$$

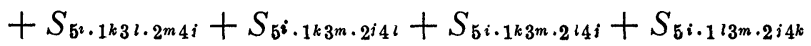

$$
\begin{aligned}
& +S_{5 i \cdot 1^{2} 3^{m \cdot 2^{k} i}}+48 \text { additional } S \text {-polynomials, }
\end{aligned}
$$


where the additional polynomials are obtained by placing $j, k, l$, and $m$ in succession as the exponent on the outstanding element, 5 .

It is to be noted that each $S$-polynomial of extent 5 can be written in five different ways. Thus

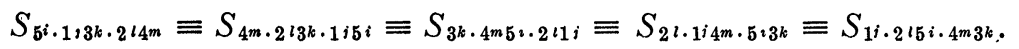

The general symmetric polynomial of extent 6 consists of the sum of $S$-polynomials of extent 6 , but since each of these is the product of some power of the elementary symmetric polynomial of degree 6 by some $S$-polynomial of extent less than 6 , they do not require further examination.

It is clear from their definitions that all the $S$-polynomials defined above belong to the simple group $G_{60}^{6}$ and that every polynomial that belongs to this group is a polynomial in the $S$-polynomials. The irreducible set that we seek is therefore to be selected from among the $S$-polynomials.

Since we may take $x_{1}, \cdots, x_{6}$ as the roots of the equation

$$
\left(x-x_{1}\right)\left(x-x_{2}\right) \cdots\left(x-x_{6}\right)=0,
$$

we can express powers of $x_{i} \geqq 6$ in terms of the elementary symmetric polynomials and powers of $x_{i}<6$. We may therefore confine our attention to $S$-polynomials for which $1 \leqq i, j, \cdots, k \leqq 6$.

The general method of procedure differs only in details from that employed in investigating the complete system of the simple group $G_{168}^{7}$ which was described in an earlier paper.* This procedure isolates a small set of polynomials from which the following 11 , forming an irreducible set, may be conveniently chosen as a complete system for the polynomials that are invariant under the simple group $G_{60}^{6}$ :

$E_{i}, \quad(i=1,2, \cdots, 6), \quad S_{123}, \quad S_{133 \cdot 24}, \quad(j=2,3,4,5)$.

VANCouver, B. C.

* On complete systems under certain finite groups, this Bulletin, vol. 37 (1931), p. 570 . 